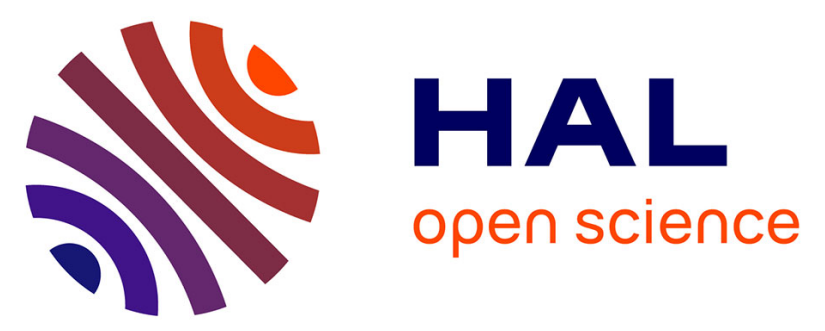

\title{
Polymer conformations and hysteretic stresses in nonstationary flows of polymer solutions
}

Nicolas François, Yacine Amarouchene, Brahim Lounis, Hamid Kellay

\section{To cite this version:}

Nicolas François, Yacine Amarouchene, Brahim Lounis, Hamid Kellay. Polymer conformations and hysteretic stresses in nonstationary flows of polymer solutions. EPL - Europhysics Letters, 2009, 86 (3), pp.34002. 10.1209/0295-5075/86/34002 . hal-00506110

\section{HAL Id: hal-00506110 https://hal.science/hal-00506110}

Submitted on 20 Dec 2017

HAL is a multi-disciplinary open access archive for the deposit and dissemination of scientific research documents, whether they are published or not. The documents may come from teaching and research institutions in France or abroad, or from public or private research centers.
L'archive ouverte pluridisciplinaire HAL, est destinée au dépôt et à la diffusion de documents scientifiques de niveau recherche, publiés ou non, émanant des établissements d'enseignement et de recherche français ou étrangers, des laboratoires publics ou privés.

\section{다(1)(2)}

Distributed under a Creative Commons Attribution - ShareAlikel 4.0 International 


\title{
Polymer conformations and hysteretic stresses in nonstationary flows of polymer solutions
}

\author{
N. François, Y. Amarouchene, B. Lounis and H. Kellay \\ Université Bordeaux 1, Centre de Physique Moléculaire Optique et Hertzienne (UMR 5798 du CNRS) \\ 351 cours de la Libération, 33405 Talence, France, EU
}

PACS 47.57.Ng - Polymers and polymer solutions

PACS $47.57 . \mathrm{Qk}$ - Rheological aspects

PACS $47.61 .-\mathrm{k}$ - Micro- and nano-scale flow phenomena

\begin{abstract}
The low Reynolds number flow of a polymer solution around a cylinder engenders a nonlinear drag force $v s$. the flow velocity. A velocity quench of such a flow gives rise to a long time relaxation and hysteresis of the stress due to history-dependent elastic effects. Our results suggest that such hysteretic behavior has its origin in the long time relaxation dynamics and hysteresis of the polymer conformations.
\end{abstract}

The conformational state of a polymer in a flow field may undergo a first- or second-order transition from a coiled to a stretched state when the deformation rate exceeds a threshold value dependent on the polymer relaxation time $[1,2]$. This was successfully confirmed by single molecule experiments and numerical simulations [3-6]. Recently, a remarkable slowdown of the relaxation of a polymer in extensional flows and in some random flows, in the vicinity of the coil-stretch transition has been found theoretically and numerically [7]. This has been attributed to hysteresis in polymer conformations as well as to the large variety in polymer conformations as observed in experiments $[3,8]$. This transition and the slowdown in relaxation have important consequences on the flow properties of polymer solutions such as the recently observed glassy-like dynamics [9] in filament stretching experiments [10]. However, few experiments have examined the link between the macroscopic phenomena and the microscopic details [11]. In this letter we aim to bridge this gap further and present both a macroscopic and a microscopic view of the transient dynamics when a polymeric solution relaxes toward a static or a dynamic stationary state by studying the interaction of a polymer-laden nonstationary flow with a cylinder embedded in it. In the stationary case, we had shown that the drag exerted on a small cylinder by a flowing polymer solution can be enhanced and is nonlinear vs. the flow velocity [12] even though the solution used has a constant shear viscosity. The nonlinearity of the stress sets in for shear rates greater than a critical shear $\dot{\gamma}_{c}$ given by the inverse of the relaxation time $\tau$ of the solution. This is characteristic of the coilstretch transition and is related to the formation of a microscopic shear band, exhibiting large molecular elongation and strong velocity fluctuations, in the immediate vicinity of the cylinder. The presence of the cylinder wall and its curvature turned out to be crucial for such large flow modifications. Here, we study a similar configuration but in the nonstationary case. The results suggest a connection between the dynamic slowdown of the macroscopic stress exerted on the obstacle and the significant hysteretic effects and slow relaxation of the polymer conformations.

We measure the stress variation upon a change of the flow velocity using our previously described optical fiber sensor and study the polymer conformations by seeding the solution with a minute amount of fluorescently labeled DNA probes [12]. The stress is measured through the deflection $d$ of the bright end of the optical fiber cantilever (radius $R=30 \mu \mathrm{m}$ ), used as a cylinder immersed in the flow, with a CCD camera working at 1000 frames per second. The force resolution of this system is $0.1 \mu \mathrm{N}$. The flow was produced in square channels of $H=6 \mathrm{~mm}$ in side and a length of $20 \mathrm{~cm}$. Using an electro-valve, we were able to create a variety of nonstationary flows with variations occurring with a characteristic time $T_{v}$ as small as $20 \mathrm{~ms}$ or as large as $1 \mathrm{~s}$. The dynamics of the deflection $d(t)$ is recorded along with the velocity variations $U(t)$ in the center of the channel where the velocity profile, in the cross section of the channel, goes through a maximum. The average velocity in the square 
channel is noted $U_{\text {mean }}(t)$, which is given by an average of the velocity profile in a cross section of the channel and is deduced from the maximum value of the velocity profile $U(t)$. In the stationary case $U_{\text {mean }}(t)$ is independent of time and is given by $Q / H^{2}$, where $Q$ is the flux through the channel. The semidilute polymer solutions use linear polyethyleneoxide (PEO, $M_{\mathrm{w}}=4 \cdot 10^{6} \mathrm{amu}$ ) that was dissolved, at a concentration of 3000 parts per million by weight $(\mathrm{ppm})$ in deionized water with $50 \%$ sugar by weight. The relaxation time $\tau$ of this solution was found to be $8 \mathrm{~ms}$. Another linear polymer (polyacrylamide (PAam)), with $M_{\mathrm{w}}=18 \cdot 10^{6} \mathrm{amu}$ and in the presence of small amounts of salt, 0 and $0.17 \mathrm{M}$, with $\tau=1.1$ and $0.06 \mathrm{~s}$, respectively), and other PEO concentrations (1500 ppm with $\tau=5 \mathrm{~ms}$ ) were also used for certain runs. The relaxation times were estimated as $\tau \simeq \frac{\left(\eta_{0}-\eta_{S}\right) M_{\mathrm{w}}}{c \rho R T}$, where $\eta_{0}$ is the zero shear viscosity of the solution, $\eta_{S}$ is the viscosity of the solvent, $R$ is the gas constant, $T$ is the temperature, $\rho$ is the density, and $c$ is the polymer concentration in ppm [13]. This time is the longest relaxation time in the system and was found comparable to relaxation times obtained from normal stress measurements, from the relaxation of the stress, and from the frequency dependence of the viscoelastic moduli of the solution. A second setup is used to infer the polymer conformations in the immediate vicinity of the cylinder in a band of about $10 \mu \mathrm{m}$ from the cylinder wall. We had previously shown that polymer elongation occurs only near the cylinder [12]. We produced $20 \mathrm{~mm}$ long, $1 \mathrm{~mm}$ wide, and $160 \mu \mathrm{m}$ high PDMS (polydimethylsiloxane) microchannels with isolated $40 \mu \mathrm{m}$ diameter and $160 \mu \mathrm{m}$ high cylindrical obstacles embedded in it. A $3000 \mathrm{ppm}$ PEO solution, in a buffer of $p \mathrm{H} 7$ and $65 \%$ by weight of added sucrose, seeded with a small amount of fluorescently labeled DNA molecules (T4-DNA of contour length $68 \mu \mathrm{m}$ and a relaxation time of $80 \mathrm{~s}$ ) was studied in this part. The relaxation time of this solution is $\tau=0.3 \mathrm{~s}$. Since the PEO molecules cannot be visualized, the best that can be done at present is to use the DNA molecules as probes of the PEO conformations even though labeled DNA can be stiffer than the nonlabeled polymers. We had previously argued that visualization of the DNA probes gives information about the conformations of the PEO molecules $[12]^{1}$. We observed the stained molecules using an inverted fluorescence microscope $(\times 60,1.45 \mathrm{NA})$ and a

\footnotetext{
${ }^{1}$ The PEO solutions showed drag enhancement above a critical $W i_{c}=\dot{\gamma}_{c} \tau$ close to 1 indicating, according to theory and experiment, that these molecules are elongated. Visualization of two different DNA molecules ( $\lambda$ and T4-DNA whose relaxation times are different from each other and much larger than that of the PEO solution) embedded in the PEO solutions showed that they are highly elongated only at or above $W i_{c}$, determined in the drag enhancement measurements, independently of the DNA used. Since the characteristic time for the transition to highly elongated DNA molecules is that of the PEO solution, which is much smaller than that of the DNA molecules, we have deduced that they are probes of the PEO conformations and that when they are elongated so are the PEO molecules and vice versa.
}

highly sensitive camera. Measurements were made at the center of depth of the channel, where the channel-induced shear rate is negligible. Inversion of the flow was performed with a system of shunting pipes and two syringe pumps. In both setups the high sucrose concentration of the solutions allows to neglect shear thinning effects.

Our optical fiber sensor, in a Newtonian fluid, is the analog of an overdamped oscillator. Its associated restoring force can be written as $F=\frac{3 \pi R^{4} E}{4 l^{3}} d$, where $E$ is the Young modulus and $l$ is the length of the fiber. In a stationary flow, the deflection of the fiber is linear vs. $U_{\text {mean }}[12]$. This remains true for the nonstationary flows used here. The deflection $d(t)$ and the velocity $U_{\text {mean }}(t)$ measured during a stop flow from a constant velocity to zero follow the same dependence vs. time. When this variation is represented in terms of stress $\sigma=\frac{F}{2 \pi R l}$ and shear rate $\dot{\gamma}=\frac{2 \cdot U_{\text {mean }}(t)}{R \cdot \ln \left(\frac{H}{2 R}-1\right)}[12,14]$, a linear relation is obtained as shown in the inset of fig. 1(a) giving a constant viscosity $\eta \sim 0.05 \mathrm{Pas}$ which compares very well with rheological measurements. This linearity confirms that fiber inertia (the fiber's characteristic time is $1 \mathrm{~ms}$ which is far below $T_{v}$ ) and transient effects inherent for a Newtonian fluid (added mass and the viscous history force) are negligible [15]. Thus, our cantilever is a viscometer that works both in the stationary and nonstationary cases.

For the polymer solutions the variation $d(t)$ and $U_{\text {mean }}(t)$ are shown in fig. 1(a) for a similar stop-flow variation as the Newtonian case discussed above. Note that, contrary to the Newtonian solution, the deflection $d(t)$ lags behind $U_{\text {mean }}(t)$. What is more striking is that even when the velocity changes sign and becomes negative, the deflection remains nonzero and positive showing a clear anti-correlation characteristic of an elastic response. A long time relaxation is also observed after the flow has been turned off. The dynamic stress exerted on the fiber turns out to be different from the stationary one, as shown in the inset. This complex response, resembling that of a yield stress fluid, exhibits both drag reduction at high shear rates and drag enhancement at lower shears with respect to the stationary case. This dynamic response actually depends on the shape of the velocity variation. For a step flow-like variation a difference between the stationary and nonstationary cases is seen only above $\dot{\gamma}_{c}$. If the velocity is varied in a range where the shear rate stays below $\dot{\gamma}_{c}, d(t)$ and $U_{\text {mean }}(t)$ follow each other giving a linear stress shear rate relation. However, when the velocity is in the range where the shear rate is higher than $\dot{\gamma}_{c}, d(t)$ and $U_{\text {mean }}(t)$ do not follow each other as shown in fig. 1(b). The full behavior of the stress strain curve from different step flows is shown in the inset of fig. 1(b) showing no effect below $\dot{\gamma}_{c}$ and strong hysteresis above it. This response is different from the one of fig. 1(a) which shows that for a stop flow, the difference with the stationary case persists even for shear rates below $\dot{\gamma}_{c}$, suggesting that history or memory effects are also important. The characteristic 

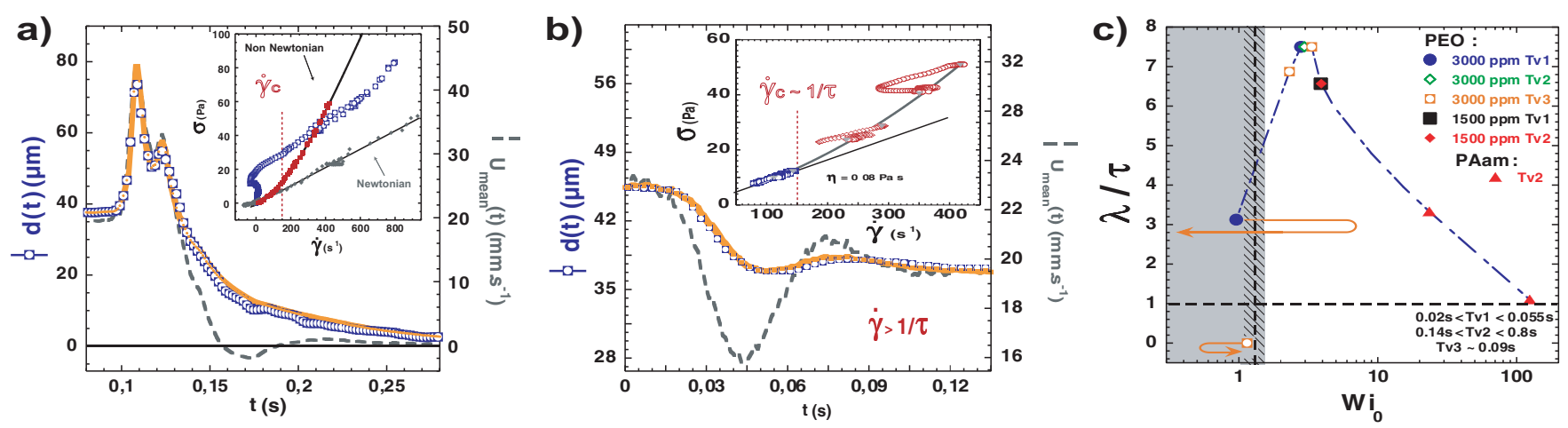

Fig. 1: (Colour on-line) Fiber deflection and velocity vs. time: (a) Polymer solution and a stop flow. (b) Polymer solution and a step flow for $\dot{\gamma}>\dot{\gamma}_{c}$. Insets: $\sigma$ vs. $\dot{\gamma}$ (open symbols) and $\sigma_{\text {stat }} v s . \dot{\gamma}$ (solid symbols and solid lines) as well as the extent of the linear regime below $\dot{\gamma}_{c}$. The Newtonian (sucrose solution) case is shown in the inset of panel (a). The solid lines in (a) and (b) are fits using our history-dependent elastic force with $\lambda=60 \mathrm{~ms}$ and $C=0.6$ in (a) and 0.78 in (b). (c) $\lambda / \tau v s . W i_{0}$ for different polymers and different flow variations, the arrows indicate the variation of $\dot{\gamma}$ and the dashed line is a guide to the eye.

closing time $T_{v}$ is much greater than $\tau$ suggesting an adiabatic change. Nonetheless, a very large difference between the dynamic stress and the stationary stress is observed. These observations are compelling evidence for the existence of an elastic history-dependent force $F_{e l}(t)$ acting on the fiber. We propose a new elastic stress $\sigma_{e l}(t)=\frac{F_{e l}(t)}{2 \pi R l_{\text {imm }}}=-\int_{0}^{t} C \cdot \exp \left(-\frac{(t-\tau)}{\lambda}\right) \frac{\partial U}{\partial \tau} \frac{\partial \sigma_{\text {stat }}}{\partial U} \mathrm{~d} \tau$, where $\sigma_{\text {stat }}$ is the stress measured in the stationary case, $C$ is a constant, and the memory function is exponential with a characteristic time $\lambda$. In fig. 1 we plot the result of the total nonstationary drag force $\sigma=\sigma_{\text {stat }}+\sigma_{e l}(t)$, which gives an accurate description for both step and stop-flow force measurements despite the large differences between them. This additional elastic force accounts for the hysteretic effects, the anti-correlation as well as the strong dependence on the flow history. Note that the clear slowdown in the dynamics introduces a long relaxation time $\lambda \sim 60 \mathrm{~ms}$ for the examples of fig. 1, a value 8 times greater than the relaxation time $\tau$. The value of $\lambda$ depends on the initial Weisenberg number $W i_{0}=\dot{\gamma}_{0} \tau,\left(\dot{\gamma}_{0}\right.$ is the initial shear rate given by the initial plateau value of the velocity) and while it is small below $W i_{c}=\dot{\gamma}_{c} \tau$, it increases as $W i_{0}$ increases, goes through a maximum near $W i_{0} \sim 3$, and decreases to values close to the relaxation time of the solution for large $W i_{0}$ as shown in fig. 1(c) where different values of $\dot{\gamma}_{0}, \tau$, and $T_{v}$ have been used. Note here that we have used data from different polymer solutions and that $\lambda$ depends on how the velocity changes in the sense that even for an initial $W i_{0}$ below threshold, if the transient shear exceeds $\dot{\gamma}_{c}$, a long time relaxation is observed. While the variation of $\lambda$ mimics the slowdown of the polymer relaxation time near the transition [7], the times measured here seem to be large for large $W i$. Collective behavior is probably important in our case considering the high concentrations used. Also, the presence of the cylinder wall and the fact that a shear band, with a large number of stretched molecules, nucleates in its vicinity [12] is another possible reason for such differences.

What is the link between such complex dynamics and the dynamics of polymer conformations? We probe the microscopic details of the polymer solution through visualizations of the polymers near the cylinder. We use the second setup to image the conformations of DNA molecules, considered as probes of the PEO conformations [12], within the PEO solution during a flow inversion. As in our previous work, we note $U_{c}$ the critical velocity to trigger the coil-stretch transition in our setup. The variation of the velocity, measured at $30 \mu \mathrm{m}$ from the cylinder (a location where the polymers are coiled), vs. time is shown in fig. 2(A). It starts out at a constant value above $U_{c}$ and goes from a positive to a negative value in a time interval of about $10 \mathrm{~s}$. The snapshots in fig. 2 display the conformations of a molecule at different positions around the cylinder taken at different times during the flow inversion. This molecule starts out as a small dot far from the cylinder (coil), starts to stretch abruptly near the upper stagnation point whose importance for the unwinding of the molecules had been stressed before [12] (images $\mathrm{a}$ and $\mathrm{b}$ ), and gets further stretched as it travels farther downstream (image c). Image $d$ is taken at the moment of inversion showing that the molecule stays stretched and remains so as it travels backward due to the flow inversion (image e). This remarkable feature was observed for a large number of molecules we have analyzed and is in agreement with previous observations [8]. The polymer conformation has not relaxed to a coil despite the long duration of the velocity inversion; the velocity stays below $U_{c}$ for roughly $2 \mathrm{~s}$ which is much longer than the relaxation time of the polymer solution. Figure 2(B) depicts the extension of a typical molecule during this flow inversion showing that it remains elongated for the full time spent at velocities below $U_{c}$; this extension is also asymmetric around the point of inversion indicating a hysteretic 

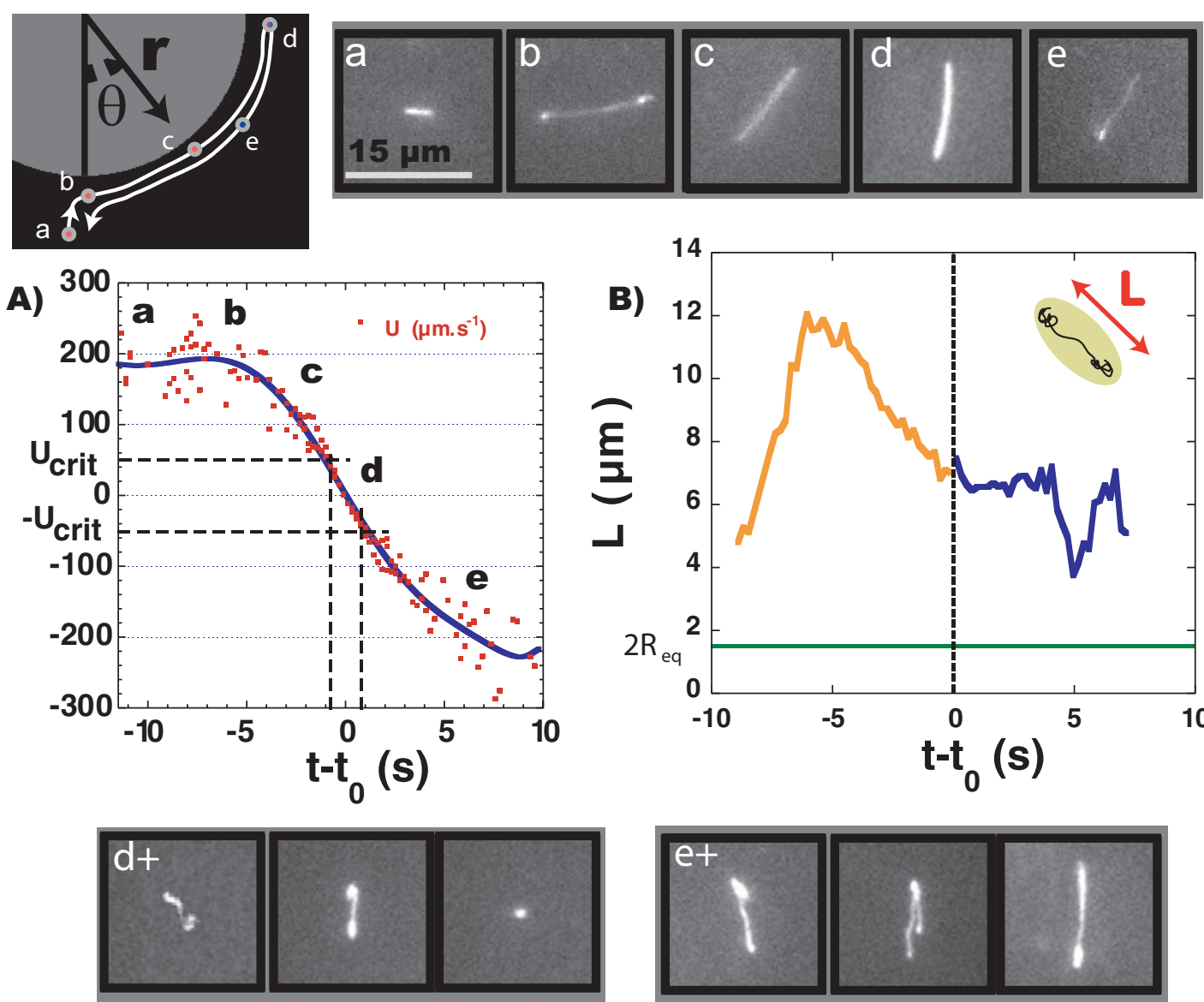
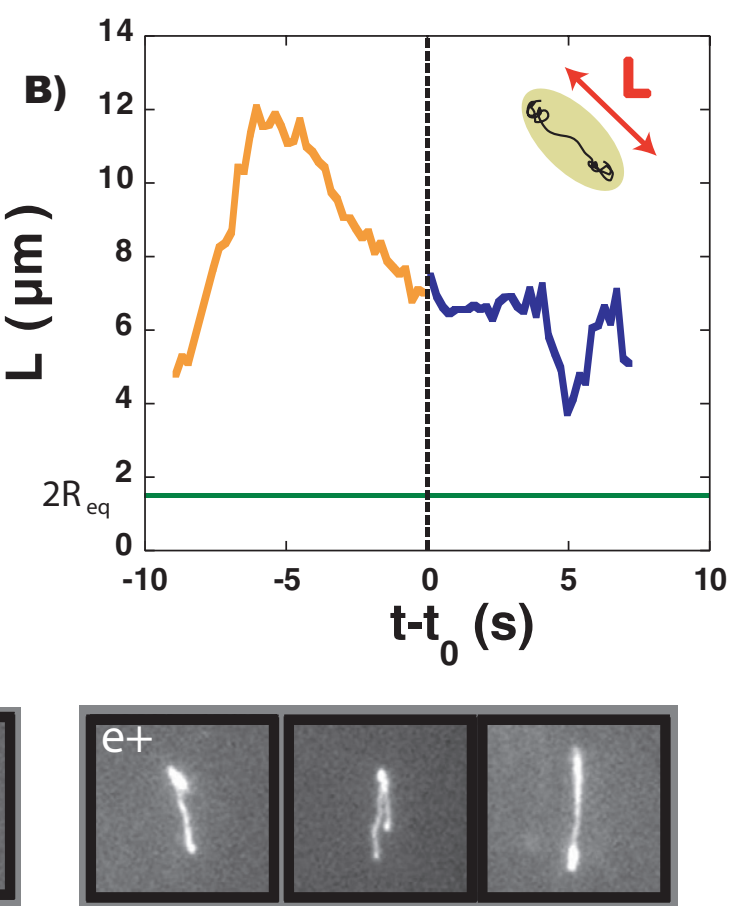

Fig. 2: (Colour on-line) T4-DNA molecules near the cylinder at different moments as shown in the schematic and indicated by letters in A which shows $U(t)$ during the flow inversion. The solid line is a guide to the eye. (B) The extension of the molecule vs. time. The photographs in $\mathrm{d}+$ and $\mathrm{e}+$ show other conformations seen at positions $\mathrm{d}$ and $\mathrm{e}$.

behavior. A variety of molecular conformations is observed during the flow inversion as shown in fig. 2 (images $d+$ and e+): simple dumbbell, "squeezed spring", folded dumbbell, stem and flower configuration, etc. showing the complexity of molecular shape relaxation [3]. Such a variety of conformations as well as the hysteresis observed are the key features of the slowdown discussed previously [7].

In the stationary flow, the extension of the molecules depends on the location $\theta$ around the cylinder and the mean velocity of the flow. In order to take such variation into account and separate the effects due to the nonstationary part of the flow from the effects observed in the stationary case, we use the ratio $\Re$ defined as: $\Re=\frac{L(\theta, t)}{\left(L_{\text {stat }}(\theta)-2 \cdot R_{\text {eq }}\right) \cdot \frac{U(t)}{U_{\text {stat }}}+2 \cdot R_{\text {eq }}}$, where $L(\theta, t)$ is the time variation of the molecular extension, $R_{e q} \sim 0.8 \mu \mathrm{m}$ is the measured equilibrium radius, and $L_{\text {stat }}(\theta)$ is the mean local extension, measured separately from experiments in the stationary case when $U=U_{\text {stat }}$. This expression takes two facts into account, first that the extension depends on the angular position $\theta$ through $L_{\text {stat }}(\theta)$ and second that this length depends on the velocity of the flow which we assume to be linear for simplicity. We plot the ratio $\Re$ in fig. 3(a) using data from 20 molecules reversing at different locations around the cylinder. Notice the large variation, from molecule to molecule, in the temporal dynamics of the extension. Despite this variety, a trend is obtained by computing the mean value of $\Re$ which is small at the high velocity end showing that the extension is similar to the stationary case far from the imposed flow variation, but increases during the flow inversion and reaches values as high as 3 or 4 showing that the molecules are much more stretched than for the stationary case. Histograms of this ratio for low velocity and high velocity values are also reported in the inset showing that a high fluctuation level exists near the flow inversion $[5,6]$ while far from it, the molecular extensions are close to their stationary state. The ratio $\Re$ is asymmetric around the flow inversion point and shows a hysteretic-like behavior. To make a link with the stress measurements, we plot in fig. 3(b) the dynamic stress of fig. 1(a) to which we subtracted the stationary stress. This curve presents a clear elastic storage at $\dot{\gamma}=0 \mathrm{~s}^{-1}$ and shows a hysteretic effect during the flow inversion. Figure 3 shows that the nonstationary part of the stress and of the molecular extension display very similar features: an asymmetry 

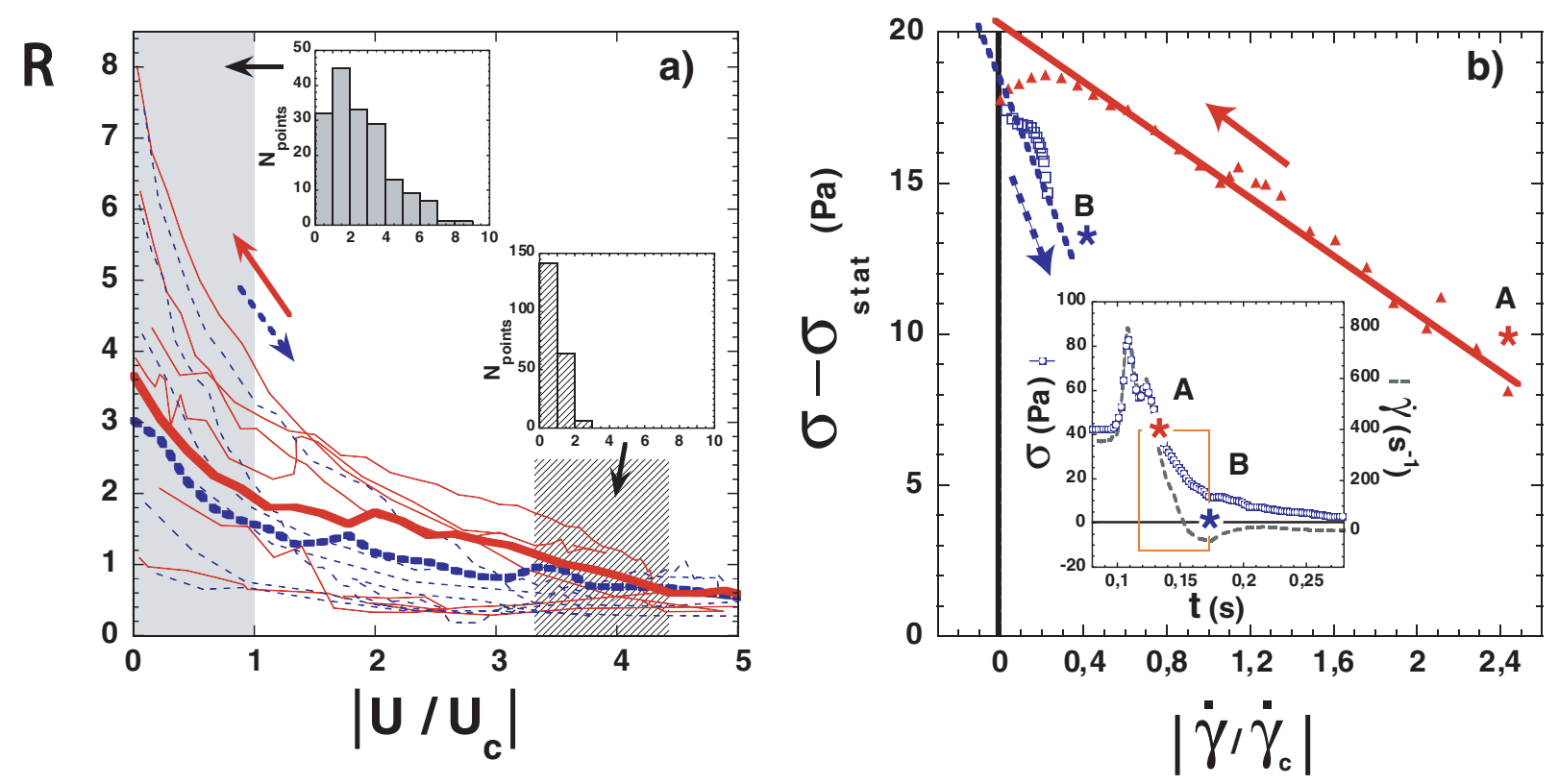

Fig. 3: (Colour on-line) (a) Nonstationary molecular extension: thin lines are for individual molecules and thick lines are for average values. (b) Excess stress vs. shear extracted from the deflection and velocity vs. time shown in the inset. The data are taken between points $A$ and $B$ where the velocity changes sign as is shown in the inset. The arrows indicate the direction of the velocity change.

around the flow inversion point and a persistent nonzero response as the flow vanishes. Since the additional stresses related to the presence of polymers are directly related to the molecular extension, we propose that the slow dynamics of the stress relaxation is related to the slow relaxation dynamics of the molecular conformations.

In conclusion, the behavior of a polymer solution in a nonstationary flow around a cylinder is dominated by a long time relaxation both for the stress and the molecular conformations. The time scale of this relaxation is much longer than the longest relaxation time of the molecules or of the solution. While slow dynamics is expected on theoretical grounds in the vicinity of the coil stretch transition, our results show that this slowdown occurs even for high deformation rates and suggest a direct link between the macroscopic response of a flowing polymer solution and the behavior of individual molecules.

\section{REFERENCES}

[1] De Gennes P. G., J. Chem. Phys., 60 (1974) 5030.

[2] Hinch E. J., Phys. Fluids, 20 (1977) S22.
[3] Sмith D. E. and Chu S., Science, 281 (1998) 1335.

[4] Perkins T. T., Smith D. E. and Chu S., Science, 276 (1997) 2016.

[5] Sмith D. E., Babcock H. P. and Chu S., Science, 283 (1999) 1724.

[6] BАвсоск H. P. et al., Macromolecules, 36 (2003) 4544.

[7] Celani A., Puliafito A. and Vicenzi D., Phys. Rev. Lett., 97 (2006) 118301.

[8] Schroeder C. M. et al., Science, 301 (2003) 1515.

[9] Sridhar T. et al., Phys. Rev. Lett., 98 (2007) 167801.

[10] McKinley G. H. and Sridhar T., Annu. Rev. Fluid Mech., 34 (2002) 375.

[11] Bавсоск H. P. et al., Phys. Rev. Lett., 85 (2000) 2018.

[12] François N. et al., Phys. Rev. Lett., 100 (2008) 18302.

[13] LaRson R. G., The Structure and Rheology of Complex Fluids (Oxford University Press) 1999; FERRY J. D., Viscoelastic Properties of Polymers (Wiley, New York) 1970.

[14] Faxìn H., Proc. R. Swed. Acad. Eng. Sci., 187 (1946) 1.

[15] Gondret P., Lance M. and Petit L., Phys. Fluids, 14 (2002) 643; Lawrence C. J. and Mei R., J. Fluid Mech., 283 (1995) 307. 